\title{
Factores de riesgo de los trabajadores de salud con COVID-19 en un hospital de Lima-Este, 2020
}

\section{Risk factors of health workers with COVID-19 in a hospital in Lima-Este, 2020}

\author{
Janett Chávez Sosa1, Brandon Gaytan Caycho²
}

\begin{abstract}
RESUMEN
Objetivo: Determinar los factores de riesgo de los trabajadores de salud con COVID-19 de un Hospital en Lima-Este. Metodologia: Estudio transversal retrospectivo de casos y controles, que consideró como caso a los trabajadores de salud con COVID-19 y cómo control a los que no tenían la infección, teniendo una muestra total de 84 trabajadores ( 28 casos y 56 controles). Los factores de riesgo que se consideraron fueron: medidas antropométricas (IMC y circunferencia abdominal), riesgo de padecer Diabetes Mellitus (Test de Findrisk) y Enfermedad Arterial Periférica (Índice tobillo/brazo). Para el análisis de datos se utilizó el paquete estadístico SPSS v.24. Para el análisis univariado, se emplearon tablas de frecuencia simple y para el bivariado tablas de contingencia con prueba de chi-cuadrado. Por último, se empleó la medida de asociación Odds Ratio (OR). Resultados: El 69\% de los trabajadores de salud fueron del sexo femenino y el $31 \%$ masculino, con una edad promedio de 44,2 $\pm 9,6$ años. La variable IMC (OR = 67,5; IC 95\% = 8,44 - 53,94) y circunferencia abdominal (OR = 6,67; IC 95\% $=1,43-31,1)$ se asociaron a un mayor riesgo de presentar COVID-19. Sin embargo, no tener el riesgo de padecer Diabetes Mellitus (OR =0,19; IC 95\% = 0,06-0,53) constituyó un factor protector para no presentar COVID-19. Conclusión: Un IMC y circunferencia abdominal elevados aumentan el riesgo de presentar COVID-19 en trabajadores de salud. En cambio, no tener un riesgo de padecer Diabetes Mellitus es un factor protector para no presentar COVID-19.
\end{abstract}

Palabras clave: Factores de riesgo, COVID-19, trabajadores de salud, pesos y medidas corporales, Diabetes Mellitus, Enfermedad Arterial Periférica (DeCS).

\begin{abstract}
Objective: To determine the risk factors of health workers with COVID-19 in a Hospital in Lima-Este. Methodology: Retrospective cross-sectional study of cases and controls, which considered health workers with COVID-19 as cases and those without infection as controls, having a total sample of 84 workers ( 28 cases and 56 controls). The risk factors that were considered were: anthropometric measurements (BMI and abdominal circumference), risk of suffering from Diabetes Mellitus (Findrisk Test) and Peripheral Arterial Disease (ankle / arm index). For data analysis, the statistical package SPSS v.24 was used. For the univariate analysis, simple frequency tables were used and contingency tables with chi-square test for the bivariate. Finally, the association measure Odds Ratio (OR) was used. Results: $69 \%$ of the health workers were female and $31 \%$ male, with an average age of 44.2 \pm 9.6 years. The variable BMI (OR $=67,5$; IC $95 \%=8,44-53,94)$ and abdominal circumference (OR $=6,67 ;$ IC $95 \%=1,43-31,1)$ were associated with a higher risk to present COVID-19. However, not having the risk of suffering from Diabetes Mellitus (OR $=0,19$; IC $95 \%=0,06-0,53$ ). It works as a
\end{abstract}

${ }^{1}$ Universidad Peruana Unión, Lima, Perú.

Orcid ID: 0000-0002-5640-5707

${ }^{2}$ Universidad Peruana Unión, Lima, Perú.

Orcid ID: 0000-0002-2654-9072 
protective factor to avoid presenting COVID-19. Conclusion: A BMI and abdominal circumference increased the risk of presenting COVID-19 in health workers. On the other hand, not having a risk of suffering from Diabetes Mellitus is a protective factor for not presenting COVID-19.

Keywords: Risk Factors, COVID-19, health workers, Body Weights and Measurements, Diabetes Mellitus, Peripheral Artery Disease (MeSH).

\section{INTRODUCCIÓN}

La Organización Mundial de la Salud (OMS, 2020), reporta 39,5 millones de casos confirmados y 1,11 millones de muertes por coronavirus (COVID-19) en el mundo (17/10/20). De igual manera la Organización Panamericana de la Salud (OPS, 2020a), informa que en América Latina y el Caribe existen 9876651 casos, con un total de 600000 muertos. El Perú ocupa el cuarto lugar dentro de los países con mayor incidencia de esta enfermedad, con un total de aproximadamente 860 mil casos y 33,577 muerto hasta la fecha (MINSA, 2020).

En la lucha contra esta enfermedad, el personal de salud ha adquirido un papel protagónico, así como un mayor riesgo de contagio. En una serie de casos temprana de Wuhan-China, el $29 \%$ de los pacientes con COVID-19 fueron trabajadores de la salud y se asumió que habían adquirido la infección en el hospital (Wang et al. 2020). Del mismo modo, en Italia, se estima que el $20 \%$ de todos los trabajadores de salud que tratan con pacientes con COVID-19 están infectados positivamente. Por otro lado, en la Región de las Américas, se tiene la mayor tasa de contagios del mundo, casi 570000 trabajadores de la salud se han enfermado y más de 2500 han fallecido, dentro de los cuales Estados Unidos y México suman casi el $85 \%$ de todas las muertes por COVID-19 de estos profesionales en la región (OPS, 2020b).

En el Perú hasta la fecha se ha informado que un total de 3,637 profesionales de la salud se han contagiado del COVID-19, y que alrededor de 165 han fallecido (Centro Nacional de Epidemiología, 2020).

El riesgo de desarrollar una infección grave por COVID-19 aumenta en personas de edad avanzada y en aquellos con condiciones de salud subyacentes (enfermedad cardiovascular, renal, respiratoria, hepática, diabetes, entre otros). De acuerdo al departamento de Epidemiología del Hospital en estudio, a través de su Programa de Prevención y Vigilancia de Enfermedades Transmisibles y No Transmisibles (PVETNT), reportó que, en febrero del 2020 el 52,33\% de los trabajadores de salud presentaron sobrepeso y el $19,73 \%$ obesidad. De igual manera, casi el $50 \%$ tuvieren un riesgo de leve a moderado de padecer diabetes mellitus y el $49 \%$ presentaban hábitos alimenticios no saludables. Estos antecedentes constituyen factores de riesgo importantes para el desarrollo de la forma grave del COVID-19. Por lo tanto, el estudio pretende estimar si estos factores de riesgo pueden ayudar en el diseño de posibles estrategias de protección, manejo y cuidado de las condiciones crónicas. Así también, a predecir una peor evolución desde el mismo momento de la infección, que puede ser útil para identificar a aquellos que deberían ser hospitalizados, o en los que hay que intensificar las medidas de soporte o iniciar precozmente los tratamientos recomendados (Martos et al. 2020).

En este estudio, investigamos la asociación entre los factores de riesgo (medidas antropométricas, riesgo de padecer Diabetes Mellitus y Enfermedad Arterial Periférica) en trabajadores de salud con COVID-19, en un hospital de Lima-Este.

\section{METODOLOGÍA}

Estudio transversal retrospectivo de casos y controles, que se llevó a cabo en trabajadores de salud que pertenecieran al Programa de Prevención y Vigilancia de Enfermedades Transmisibles y No Transmisibles (PVETNT) del Hospital de Huaycán II-1, Lima, Perú. La población total de trabajadores que pertenecen a dicho programa, es de 316 , de los cuales 214 cumplían función asistencial.

Para la selección de los casos, se empleó el muestreo no probabilístico y por conveniencia, a través de la base de datos brindada por el área de Epidemiología del Hospital, informando un 
total de 130 trabajadores diagnosticados con COVID-19 hasta el 17/10/2020, de los cuales 48 pertenecían al Programa de PVETNT y 28 tenían los datos completos. La muestra final estuvo conformada por 84 trabajadores de salud: 28 casos y 56 controles.

Dentro de la base de datos, se seleccionaron como factores de riesgo a las: medidas antropométricas (IMC y circunferencia abdominal), el riesgo de padecer Diabetes Mellitus (Test de Findrisk) y la Enfermedad Arterial Periférica (Índice tobillo/brazo). Las variables fueron dicotomizadas de acuerdo a los siguientes valores: IMC $\left(\mathrm{Kg} / \mathrm{m}^{2}\right)$ normal de 18,5 - 24,9 y aumentado $\geq 25$; circunferencia abdominal normal $\leq 88 \mathrm{~cm}$ en mujeres $\mathrm{y} \leq 102$ $\mathrm{cm}$ en hombres (Pajuelo et al. 2019); Test de Findrisk de 0-7 puntos bajo riesgo de padecer Diabetes Mellitus y $>7$ alto riesgo; y por último, el índice tobillo/brazo, con valores de 0,9-1,3 sin Enfermedad Arterial Periférica y $<0,9$ con Enfermedad Arterial Periférica.

Para el análisis de datos se usó el Programa estadístico SPSS v.24. Se utilizaron tablas de frecuencia simple para las variables categóricas y medidas de tendencia central (media aritmética) y dispersión (desviación estándar) para las variables numéricas. Se emplearon tablas de contingencia de doble entrada para ver la asociación entre variables con la prueba estadística chi-cuadrado. Asimismo, se halló el Odds Ratio (OR) para determinar los factores de riesgo para COVID-19 en trabajadores de salud.

\section{RESULTADOS}

En la tabla 1 se observa que la edad promedio de los trabajadores de salud fue de 44,2 años, entrando en la categoría de adulto; asimismo, el $69 \%$ fueron del sexo femenino y el $31 \%$ masculino. En torno a las medidas antropométricas, se obtuvo un peso promedio de $63,6 \pm 2,9 \mathrm{~kg}$ con una talla de 1,55 $\pm 0,08 \mathrm{~cm}$. Al cálculo del IMC el $51,2 \%$ de los trabajadores lo presentó elevado y se obtuvo una circunferencia abdominal de $84,7 \pm 9,9 \mathrm{~cm}$. Por otro lado, el $71,4 \%$ presentaron EAP y el $72,6 \%$ tuvo riesgo de padecer DM en el futuro.

Tabla 1

Análisis descriptivo de la población de estudio.

\begin{tabular}{|c|c|c|c|}
\hline Variables & & $n=84$ & $\%$ \\
\hline Edad & $\mathrm{Me} \pm \mathrm{DS}$ & $44,2 \pm 9,6$ & \\
\hline \multirow[t]{2}{*}{ Sexo } & Femenino & 58 & 69,0 \\
\hline & Masculino & 26 & 31,0 \\
\hline Peso & $\mathrm{Me} \pm \mathrm{DS}$ & $63,6 \pm 2,9$ & \\
\hline Talla & $\mathrm{Me} \pm \mathrm{DS}$ & $1,55 \pm 0,08$ & \\
\hline \multirow[t]{2}{*}{ IMC } & Normal & 41 & 48,8 \\
\hline & Aumentado & 43 & 51,2 \\
\hline Circunferencia abdominal & $\mathrm{Me} \pm \mathrm{DS}$ & $84,7 \pm 9,9$ & \\
\hline \multirow[t]{2}{*}{ EAP } & Sí & 60 & 71,4 \\
\hline & No & 24 & 28,6 \\
\hline \multirow[t]{2}{*}{ Riesgo de padecer DM } & Sí & 61 & 72,6 \\
\hline & No & 23 & 27,4 \\
\hline
\end{tabular}

*IMC: índice de masa corporal; EAP: Enfermedad Arterial Periférica; Me: Media aritmética; DS: desviación estándar. 
Seguidamente, en la tabla 2 se infiere que la variable IMC (OR $=67,5$; IC $95 \%=8,4-53,9)$ y circunferencia abdominal (OR $=6,6$; IC $95 \%=1,4-31,4)$ se asociaron a un mayor riesgo de presentar COVID-19. Es decir, los trabajadores de salud con un IMC y circunferencia abdominal aumentado tuvieron 67,5 y 6,6 veces mayor riesgo de padecer COVID-19, respectivamente. En cambio, no tener el riesgo de padecer Diabetes Mellitus (OR = 0,19; IC 95\% = 0,06-0,53) constituyó un factor protector para no presentar COVID-19.

Tabla2

Factores de riesgo asociados a COVID-19 en trabajadores de salud de un hospital de Lima-Este, 2020.

\begin{tabular}{|c|c|c|c|c|c|c|c|c|}
\hline \multirow{2}{*}{ Factores de riesgo } & & & \multirow{2}{*}{ Total } & \multicolumn{2}{|c|}{ COVID-19 } & \multirow{2}{*}{ OR } & \multirow{2}{*}{ IC $95 \%$} & \multirow{2}{*}{ p-valor } \\
\hline & & & & Sí & No & & & \\
\hline \multirow[t]{4}{*}{ Sexo } & Femenino & $\mathrm{n}$ & 58 & 19 & 39 & \multirow{4}{*}{1,08} & \multirow{4}{*}{$\begin{array}{l}0,40- \\
2,88\end{array}$} & \multirow{4}{*}{0,867} \\
\hline & & $\%$ & $100,0 \%$ & $32,8 \%$ & $67,2 \%$ & & & \\
\hline & Masculino & $n$ & 26 & 9 & 17 & & & \\
\hline & & $\%$ & $100,0 \%$ & $34,6 \%$ & $65,4 \%$ & & & \\
\hline \multirow[t]{4}{*}{ Edad } & Adulto & $n$ & 80 & 27 & 53 & \multirow{4}{*}{0,65} & \multirow{4}{*}{$\begin{array}{l}0,06- \\
6,59\end{array}$} & \multirow{4}{*}{0,499} \\
\hline & & $\%$ & $100,0 \%$ & $33,8 \%$ & $66,3 \%$ & & & \\
\hline & Adulto mayor & $n$ & 4 & 1 & 3 & & & \\
\hline & & $\%$ & $100,0 \%$ & $25,0 \%$ & $75,0 \%$ & & & \\
\hline \multirow[t]{4}{*}{ IMC } & Normal & $n$ & 41 & 1 & 40 & \multirow{4}{*}{67,5} & \multirow{4}{*}{$\begin{array}{l}8,44- \\
53,9\end{array}$} & \multirow{4}{*}{0,000} \\
\hline & & $\%$ & $100,0 \%$ & $2,4 \%$ & $97,6 \%$ & & & \\
\hline & Aumentado & $n$ & 43 & 27 & 16 & & & \\
\hline & & $\%$ & $100,0 \%$ & $62,8 \%$ & $37,2 \%$ & & & \\
\hline \multirow{4}{*}{$\begin{array}{l}\text { Circunferencia } \\
\text { abdominal }\end{array}$} & Normal & $n$ & 21 & 2 & 19 & \multirow{4}{*}{6,67} & \multirow{4}{*}{$\begin{array}{l}1,43- \\
31,1\end{array}$} & \multirow{4}{*}{0,008} \\
\hline & & $\%$ & $100,0 \%$ & $9,5 \%$ & $90,5 \%$ & & & \\
\hline & Aumentado & $n$ & 63 & 26 & 37 & & & \\
\hline & & $\%$ & $100,0 \%$ & $41,3 \%$ & $58,7 \%$ & & & \\
\hline \multirow[t]{4}{*}{ EAP } & No & $n$ & 24 & 7 & 17 & \multirow{4}{*}{1,30} & \multirow{4}{*}{$\begin{array}{l}0,46- \\
3,65\end{array}$} & \multirow{4}{*}{0,608} \\
\hline & & $\%$ & $100,0 \%$ & $29,2 \%$ & $70,8 \%$ & & & \\
\hline & Sí & $n$ & 60 & 21 & 39 & & & \\
\hline & & $\%$ & $100,0 \%$ & $35,0 \%$ & $65,0 \%$ & & & \\
\hline \multirow{4}{*}{$\begin{array}{l}\text { Riesgo de padecer } \\
\text { DM }\end{array}$} & No & $n$ & 23 & 14 & 9 & \multirow{4}{*}{0,19} & \multirow{4}{*}{$\begin{array}{l}0,06- \\
0,53\end{array}$} & \multirow{4}{*}{0,002} \\
\hline & & $\%$ & $100,0 \%$ & $60,9 \%$ & $39,1 \%$ & & & \\
\hline & Sí & $n$ & 61 & 14 & 47 & & & \\
\hline & & $\%$ & $100,0 \%$ & $23,0 \%$ & $77,0 \%$ & & & \\
\hline
\end{tabular}

*IMC: índice de masa corporal; EAP: Enfermedad Arterial Periférica; DM: Diabetes Mellitus; Me: Media aritmética; DS: desviación estándar, IC: Intervalo de confianza; OR: Odds Ratio

\section{DISCUSIÓN}

El COVID-19 ha cogido por sorpresa a los sistemas de salud de todo el mundo, asimismo, la falta de guías de atención genera incertidumbre al momento de tratar la enfermedad. Se estima que alrededor del $1 \%$ de la población mundial expresa la forma grave de la enfermedad, lo que ha generado el desborde del sector sanitario. Por otro lado, estudios evidencian que los trabajadores de salud son unas de las poblaciones más susceptibles al contagio debido al contacto directo con estos pacientes, lo que hace necesario conocer los factores de riesgo que lo predisponen a mayor mortalidad por la enfermedad (Córdova y Rossani, 2020). 
Elestudioencontróquelamayoría detrabajadores de salud eran del sexo femenino y tenían una edad promedio de 44,2 años. Asimismo, presentaron IMC y circunferencia abdominal aumentados, tuvieron riesgo de padecer DM2 y presentaron EAP (tabla 1). Las variables que se asociaron significativamente con la presencia de COVID-19 en trabajadores de salud fueron el IMC y la circunferencia abdominal (tabla 2). A la revisión de la literatura, no se hallaron estudios sobre obesidad y COVID-19 en trabajadores de salud, sin embargo, una revisión sistemática de artículos en idioma chino e inglés, llevado a cabo por Barry y colaboradores (2020) en población general obesa, reveló que las personas con obesidad tenían un mayor riesgo de COVID-19 positivo; tanto para la hospitalización, ingreso a $\mathrm{UCl}$ y alta mortalidad.

La literatura señala que existen 2 factores pronósticos de alta mortalidad en pacientes con infección por COVID-19, estos son: los valores de IMC > $25 \mathrm{Kg} / \mathrm{m} 2$ tanto en hombres como mujeres y una circunferencia abdominal $>102$ $\mathrm{cm}$ en hombres y $>88 \mathrm{~cm}$ en mujeres (Enguita, Corroza y Ostolaza, 2020).

Esto en gran parte se debe a que, a mayor grasa abdominal, el diafragma asciende comprimiendo el parénquima pulmonar, causando una obstrucción al flujo de aire que ingresa a los pulmones; que conduce al colapso de las vías respiratorias en los lóbulos inferiores de los pulmones y condiciona el empeoramiento del cuadro clínico del paciente (Rivera, Fornaris, Ledesma, López y Pérez, 2018). Además de ello, la sangre de las personas con sobrepeso o circunferencia abdominal aumentada, tienden a presentar estados de hipercoagulabilidad sobre todo a nivel microvascular, que ocasionarían la aparición de trombos rápidamente, llevando a un empeoramiento del cuadro clínico (Fiestas, Peralta, Zavala y Barrionuevo, 2020; Rivera, Fornaris, Ledesma, López y Pérez, 2018). De igual manera, el sistema inmune en pacientes obesos también se ve alterado, debido a la pérdida de tejido inmunológico que es reemplazado por tejido adiposo, lo que disminuye el número de células $T$ de memoria que aumentan el riesgo de reinfección (Petrova et al. 2020).

Otro resultado importante del estudio, demostró que tener un bajo riesgo de padecer DM actúa como un factor protector para la infección por COVID-19 en trabajadores de salud. Según
Figueroa y colaboradores (2020), los pacientes con diabetes tienen un mayor riesgo de desarrollar complicaciones graves durante el curso de la enfermedad como: el síndrome de dificultad respiratoria del adulto y la insuficiencia multiorgánica. Esto debido, a dos mecanismos específicos: primero, se ha demostrado que la hiperglucemia eleva la expresión de la enzima convertidora de angiotensina 2 (ACE2) en las células, lo que podría facilitar la entrada de células virales; y segundo a través de la enzima inhibidora de dipeptidil peptidasa-4 (DPP-4) que actuaría como un receptor funcional para la virulencia por COVID 19 (Cajamarca et al. 2020; López y Herrera, 2020).

\section{Declaración de financiamiento y de conflictos de interés}

El estudio fue financiado por los autores, quienes declaran no tener algún tipo de conflicto de interés en la investigación realizada.

\section{Correspondencia}

Janett Chávez Sosa

Correo electrónico:

viki16@upeu.edu.pe

Brandon Gaytan Caycho

Correo electrónico:

brandongaytan@upeu.edu.pe

\section{REFERENCIAS BIBLIOGRÁFICAS}

Barry M, Shufa D, Sheekar M, 2020. "Individuals with obesity and COVID-19: A global perspective on the epidemiology and biological relationships". Obesity Reviews 21(11): 1-17.

Córdova A, Rossani A. 2020. "COVID-19: Literature Review and Its Impact on the Peruvian Health Reality." Revista de la Facultad de Medicina Humana 20(3): 467-73.

Cajamarca J, Cubides H, Guavita D, Buitrago J, Gallego L, Navas A, Arredondo A, Escobar A, Rojas A, 2020. SARS-CoV-2 (COVID-19) en pacientes con algún grado de inmunosupresión. Reumatología Clínica 37(5): 1-12. 
Centro Nacional de Epidemiología, Prevención y Control de Enfermedades. 2020. "CDC MINSA - Centro Nacional de Epidemiología, Prevención y Control de Enfermedades." 23/07. https://www. dge.gob.pe/portalnuevo/ (June 6, 2021).

Martos J. Luque J. Jiménez E, Mora B, Núnez C, Asencio A, García J , Navarro F. 2020. "Comorbidity and Prognostic Factors on Admission in a COVID-19 Cohort of a General Hospital." Revista Clínica Española . DOI: 10.1016/j.rce2020.05.017

Fiestas F; Peralta V; Zavala J; Barrionuevo P. 2020. letsi - Essalud Obesidad Como Factor De Riesgo Para Covid 19. Lima. http://www.essalud.gob.pe/ ietsi/pdfs/covid_19/RB_36_Obesidad_30_jul_20.

Figueroa J, Salas A, Buitrago A. 2020. "COVID-19 and Cardiovascular Disease." Revista Colombiana de Cardiologia 27(3): 166-74. DOI:10.1016/j. rccar.2020.04.004.

Enguita J, Corroza J, Ostolaza A. 2020. "Factores de Riesgo y Predictores de Gravedad En Pacientes Hospitalizados Por COVID-19: Análisis de 52 Casos." Medicina Clínica 155(8): 360-61.

Organización Mundial de la Salud. 2020. "WHO Coronavirus (COVID-19) Dashboard | WHO Coronavirus (COVID-19) Dashboard With Vaccination Data." 17/10. https://covid19.who.int/ (June 6, 2021).

Organización Panamericana de la Salud. 2020a. "Brote de Enfermedad Por El Coronavirus [(COVID-19)" OPS/OMS | Organización Panamericana de La Salud." 28/06. https://www.paho.org/es/temas/ coronavirus/brote-enfermedad-por-coronaviruscovid-19 (June 6, 2021).

Organización Panamericana de la Salud. 2020b. "Cerca de 570.000 Trabajadores de La Salud Se Han Infectado y 2.500 Han Muerto Por COVID-19 En Las Américas - OPS/OMS | Organización Panamericana de La Salud." 02/09. https://www. paho.org/es/noticias/2-9-2020-cerca-570000- trabajadores-salud-se-han-infectado-2500-hanmuerto-por-covid-19 (June 6, 2021).

Pajuelo J, Torres L, Agüero R, Bernui I. Sobrepeso y obesidad en la población adulta del Perú. An Fac med. 2019;80(1):21-7. DOI: 10.15381/ anales. v80i1.15863.

Petrova D, Salamanca E, Rodríguez M, Navarro P, Jiménez J, Sánchez M. 2020. "Obesity as a Risk Factor in COVID-19: Possible Mechanisms and Implications." Atencion Primaria 52(7): 496-500. DOI: 10.1016/j.aprim.2020.05.003.

Rivera E, Ledesma A, López M, Pérez G. 2018. "Circunferencia Abdominal y Riesgo de Enfermedades Cardiovasculares. Consultorio 22. Policlìnico Docente 'Aleida Fernàndez Chardiet'. 2016." Revista Habanera de Ciencias Médicas 17(4): 12.

Ministerio de Salud. 2020. "MINSA: Casos Confirmados Por Coronavirus Covid-19 Ascienden a 371,096 En El Perú (Comunicado № 181) | Gobierno Del Perú." 23/07. https:// www.gob.pe/institucion/minsa/noticias/215539minsa-casos-confirmados-por-coronaviruscovid-19-ascienden-a-371-096-en-el-perucomunicado-n-181 (June 6, 2021).

Wang D, Hu B, Chang H, Fangganf Z, Xing L, Jing Z, Binbin W, Hui X, Zhenshun C, Yong X, Yan Z, Yirong L, Xinghuan W, Zhiyong P. 2020. "Clinical Characteristics of 138 Hospitalized Patients with 2019 Novel Coronavirus-Infected Pneumonia in Wuhan, China." JAMA - Journal of the American Medical Association 323(11): 1061-69. DOI: 10.10101/jama.2020.1585

Recibido: 07/02/2021

Aceptado: 30/04/2021 